\title{
EDITORIAL
}

\section{LA ELIMINACIÓN DE LA SÍFILIS CONGÉNITA EN COLOMBIA: UNA UTOPÍA SI NO SE APLICAN LAS MEDIDAS DE CONTROL}

\author{
JORGE ENRIQUE PÉREZ CARDENAS
}

En la XXIV Conferencia Sanitaria Panamericana, realizada en 1994, Colombia se comprometió a alcanzar la meta de 0,5 casos de sífilis congénita por cada 1000 nacidos vivos antes del año 2000. A la fecha esta meta no se ha logrado, lo cual indica que las medidas implementadas para controlar este problema no han sido lo suficientemente efectivas. La incidencia ha disminuido año tras año; sin embargo, debido al cambio de las guías de práctica clínica en el 2015 y al uso de un algoritmo diferente en el diagnóstico de la sífilis por el laboratorio, estas cifras pueden volver a aumentar.

La sífilis congénita se considera un problema de salud pública debido a la generación de muerte fetal y neonatal, asociada a las secuelas que produce y de acuerdo con el momento en que se adquiere la infección. Esta enfermedad rara vez se adquiere antes del cuarto mes de embarazo, por lo que es raro encontrar abortos relacionados con la infección durante este periodo (1).

El acceso de las mujeres embarazadas al sistema de salud es un factor importante en la disminución de los casos de la sífilis congénita, pues la mayoría de las mujeres con sífilis gestacional provienen del régimen subsidiado. El tratamiento de la sífilis en mujeres embarazadas con sospecha o confirmación de la enfermedad, no garantiza la posibilidad de que el feto se infecte (1). Por esto es importante la realización de pruebas treponémicas y no treponémicas en recién nacidos asintomáticos o con manifestaciones compatibles con sífilis, y es necesario hacer seguimiento a las mujeres con enfermedad gestacional para comprobar la efectividad del tratamiento.

Desde finales de 2010 el Ministerio de Protección Social, hoy Ministerio de Salud y Protección Social, hizo un diagnóstico de las razones por las que hasta el año 2009 se presentaba una incidencia nacional de la infección congénita de 1,26 casos por 1000 nacidos vivos. Se encontró que, de 23370 mujeres embarazadas infectadas en 2010, nacieron 16602 niños con sífilis congénita (2); esto implica que del $71 \%$ de las mujeres embarazadas infectadas nacen niños con la enfermedad o que el número de casos de sífilis gestacional es más alto de lo reportado. Sin embargo, según este informe, el Departamento Administrativo de Estadística (DANE) reportó que para 2005, el $94 \%$ de las mujeres en embarazo acudieron a control prenatal, lo que implica probables fallas en la atención de las gestantes (2).

Esta alta tasa de infección en los bebés es posible que haya disminuido a la fecha. Sin embargo, la cobertura no es adecuada si se tiene en cuenta que la incidencia a la semana 39 de este año es de 0,70 casos por 1000 nacidos vivos (3), valores inferiores a los reportados en 2010, mostrando la tendencia de presentación de menos casos cada año, pero a una velocidad de disminución baja que indica que las medidas tendientes a resolver la situación se cumplen en unas localidades del país y en otras no, y que hacen falta medidas más proactivas por parte de los entes institucionales asociados con la salud pública, tendientes a alcanzar una meta de cero casos de sífilis neonatal.

\footnotetext{
${ }^{1}$ Profesor, Departamento de Ciencias Básicas. Facultad de Ciencias para la Salud. Universidad de Caldas
} 
Este evento es casi imposible si se tiene en cuenta que, a pesar de existir protocolos bien establecidos para la detección y manejo de la sífilis gestacional y congénita, posiblemente no se cumplen a cabalidad, en especial en lo concerniente al tratamiento de parejas sexuales que pueden reinfectar a la mujer embarazada y de esta manera mantener o aumentar el riesgo de infección neonatal. En este aspecto, es importante que las gestantes sigan asistiendo a su control prenatal para detectar una posible reinfección. Es posible que aquí se presenten fallas para la consecución de la meta, ya que posiblemente no se hace una búsqueda de estas mujeres para que vuelvan a su control prenatal y solamente aparecen en el momento del parto, cuando la sífilis ya está en el recién nacido.

Por otro lado, a pesar de que es posible que haya mejorado el registro, en algunas zonas del país se sigue presentado subregistro, que estaría demostrando que los casos de sífilis congénita pueden ser mayores a los reportados, y a la vez, impide la implementación de las medidas correctivas por parte de las autoridades de salud pública para mejorar la cobertura de atención de las embarazadas y así controlar esta enfermedad. Además, la baja cobertura rural podría ser otro factor que ha evitado que las metas del control de la sífilis se cumplan.

Se requiere entonces que las entidades territoriales de salud intensifiquen sus mecanismos de reporte y control de la enfermedad, inicialmente presionando a las secretarías de salud de los municipios en los que se observa mayor incidencia de la enfermedad, para que inicien campañas que motiven a las mujeres embarazadas a ir a su control prenatal, hagan brigadas rurales de salud para mejorar el acceso a las consultas prenatales; ofrezcan los medios de diagnóstico necesarios para la búsqueda de mujeres infectadas, y hagan un adecuado control y seguimiento de las mujeres infectadas pos tratamiento, para evitar o detectar reinfecciones o recaídas asociadas con un tratamiento inadecuado. También se requiere que se cumpla localmente con la notificación semanal de casos de sífilis gestacional y congénita. Estos dos factores podrían contribuir de manera significativa a disminuir los casos de sífilis congénita en los recién nacidos de nuestro país.

\section{BIBLIOGRAFÍA}

1. Tramont EC. Treponema pallidum (Syphilis). In: Mandell GL, Bennett JE, Dolin R. eds. Principles and practice of infectious diseases. Philadelphia, PA. Churchill Livingston Elsevier. Seventh edition; 2010: 3035-3053.

2. Ministerio de Protección Social. Plan estratégico para eliminación de la trasmisión materno infantil del VIH y la sífilis congénita. Colombia 2011-2015.

3. Instituto Nacional de Salud. Infecciones de transmisión sexual. Boletín Epidemiológico Semanal. Semana 39, septiembre 25 al 01 de octubre; 40-43. 